\title{
Hybrid Detection Method for Improving Spectrum Sensing Performance in Cognitive Radio
}

\author{
Chinchu $\mathbf{S}^{1}$, Sangeeta $\mathbf{T} \mathbf{R}^{2}$ \\ ${ }^{1}$ M-Tech Student, Department of Communication Engineering, Sree Buddha College of Engineering for Women, Elavumthitta, Kerala, India \\ ${ }^{2}$ Assistant Professor, Department of Electronics and Communication Engineering, Sree Buddha College of Engineering for Women, \\ Elavumthitta, Kerala, India
}

\begin{abstract}
Cognitive radio is the core prominent wireless technologies for the next generation wireless communication systems. Its main feature is the capability to increase the utilization of the currently scarce spectrum resources by allowing unlicensed users to access unused licensed frequency bands opportunistically. Energy detection based spectrum sensing is the most commonly used spectrum sensing technique in cognitive radio. In order to efficiently coordinating spectrum resources, wideband multichannel spectrum utilizes fast Fourier transform for spectrum sensing. This energy detection method is inherently sensitive to noise variances and increased hardware complexity. At low signal-to-noise ratio values energy detection degrades the spectrum sensing performance. A hybrid spectrum sensing method can be effectively used to achieve better spectrum sensing performance. Hybrid spectrum sensing utilizes a combination of energy detection and Max-Min Eigen value based detection. This is a highly effective method for ensuring acceptable spectrum sensing performance. Spectrum analysis is done using fast Fourier transform.
\end{abstract}

Keywords: Cognitive radio, Spectrum sensing, Energy detection, Eigen value detection.

\section{Introduction}

Cognitive radio (CR) can successfully deal with the growing demand and scarcity of the wireless spectrum. A CR is defined by Federal Communications Commission (FCC) as "A radio or system that senses its operational electromagnetic environment and can dynamically and autonomously adjust its radio operating parameters to modify system operation, such as maximize throughput, mitigate interference, facilitate interoperability, access secondary markets." To exploit limited spectrum efficiently, CR technology allows unlicensed users to access licensed spectrum bands [1]. Since licensed users have priorities to use the bands, the unlicensed users need to continuously monitor the licensed user's activities to avoid interference and collisions. Based on the sensing results, the unlicensed users should adapt their transmit powers and access strategies to protect the licensed communications. The unlicensed users also called secondary users (SUs) need to continuously monitor the activities of the licensed users called primary users (PUs) to find the spectrum holes (SHs), which is defined as the spectrum bands that can be used by the SUs without interfering with the PUs. This is called spectrum sensing.

Spectrum sensing (SS) is the basic task of cognitive radio system which helps to make decision that whether PU is occupying a particular frequency band or not. There are different spectrum sensing schemes are used such as energy detection, matched filter detection, cyclostationary detection, maximum-minimum Eigen value detection etc. Energy detection (ED) is among the most popular spectrum sensing method. The Neyman-Pearson type binary hypothesis testing problem is commonly used to formulate ED [2]. The main shortcoming of ED is its sensitivity to the noise variance. Below an SNR threshold, energy detection is not an effective method of spectrum sensing. This low SNR is called SNR wall.
Eigen value based spectrum sensing techniques which need no noise variance information. So small changes or uncertainty on the noise variance have only a small effect on the sensing performance [3]. The changes are just due to changes in SNR, if PU signal power is assumed to be constant. In order to improve spectrum sensing performance a combination of energy detection and Eigen value based detection schemes are used. These two sensing schemes are based on threshold calculation of the received signal. The threshold based detectors are used to improve sensing performance.

\section{Hybrid Detection Method}

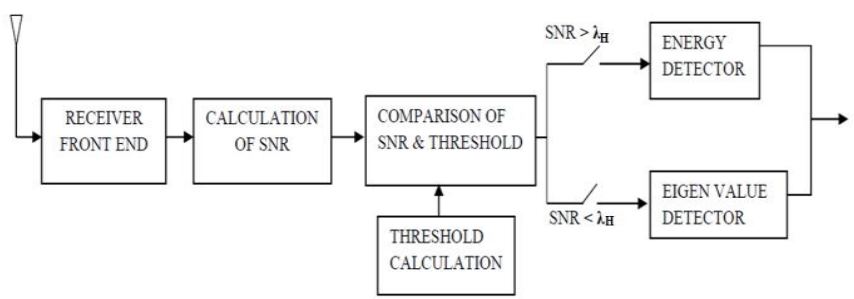

Figure 1: Block diagram of hybrid detection method

A new hybrid spectrum sensing scheme which involves a FFT based energy detector and max-min Eigen value detector is proposed.

Figure 1 shows the hybrid spectrum sensing method. The received signal SNR level value is compare with the threshold value. If SNR value is greater than the threshold value, energy detection method can be used for spectrum sensing. When SNR is less than the threshold, maximum-minimum Eigen value detector can be used. The energy detection is not an effective method due to the existence of SNR wall or high 


\section{International Journal of Science and Research (IJSR) \\ ISSN (Online): 2319-7064}

Index Copernicus Value (2013): 6.14 | Impact Factor (2015): 6.391

probability of false alarm under noise uncertainty condition. High noise variance causes degradation of spectrum sensing performance. This threshold based detector will improve the spectrum sensing performance in cognitive radio.

\subsection{Energy Detector}

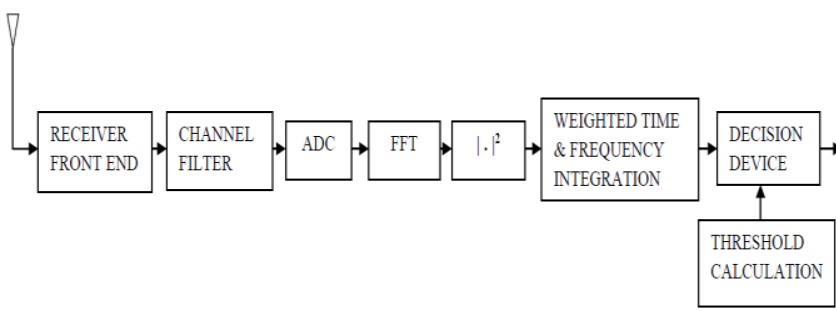

Figure 2: Block diagram of energy detector

The basic block diagram of ED with FFT based spectrum analysis is shown in figure 2. After the receiver front-end, channel filter and analog to digital converter (ADC), FFT is employed to split the signal into comparatively narrow frequency bands. The channel filter selects the frequency bands which is basically a band pass filter. The output data blocks can be expressed as $\mathrm{y}_{\mathrm{k}}[\mathrm{m}]$, where $k$ is the sub band index and $m$ is the sub band sample index. It is assumed that the sampling rate used in each sub band is equal to the ADC sampling rate divided by the number of sub bands in the filter bank. The sub bands signals can be expressed as,

$$
\begin{gathered}
\mathrm{y}_{\mathrm{k}}[\mathrm{m}]=\mathrm{w}_{\mathrm{k}}[\mathrm{m}] \mathrm{H}_{0} \\
\mathrm{y}_{\mathrm{k}}[\mathrm{m}]=\mathrm{x}_{\mathrm{k}}[\mathrm{m}]+\mathrm{w}_{\mathrm{k}}[\mathrm{m}] \mathrm{H}_{1}
\end{gathered}
$$

Where $x_{k}[m]$ is the primary user information signal at the $m^{\text {th }}$ FFT output sample in sub band $\mathrm{k}$, and $\mathrm{w}_{\mathrm{k}}[\mathrm{m}]$ is the corresponding noise sample. The integrated test statistic over multiple sub bands is,

$$
T\left(y_{m_{0}, k_{0}}\right)=\frac{1}{N_{t} N_{f}} \sum_{k=k_{0}-\left[N_{f} / 2\right]}^{k_{0}+\left[N_{f} / 2\right]-1} \sum_{m=m_{0}-N_{t}+1}^{m_{0}}\left|y_{k}[m]\right|^{2}
$$

Where $\mathrm{N}_{\mathrm{f}}$ and $\mathrm{Nt}$ are the averaging filter lengths in frequency domain and time domain. The energy detection requires no prior information about the primary user, signal is detected by comparing the measured energy with the threshold value. The performance of energy detector depends on probability of false alarm and probability of detection.

$$
\begin{gathered}
P_{F A}=\operatorname{Pr}\left(T(y)>\lambda \mid \mathcal{H}_{0}\right)=Q\left(\frac{\lambda-\sigma_{w, k}^{2}}{\sigma_{w, k}^{2} / \sqrt{N_{f} N_{t}}}\right) \\
P_{D}=\operatorname{Pr}\left(T(y)>\lambda \mid \mathcal{H}_{1}\right)=Q\left(\frac{\lambda-\sigma_{w, k}^{2}\left(1+\gamma_{k}\right)}{\sigma_{w, k}^{2}\left(1+\gamma_{k}\right) / \sqrt{N_{f} N_{t}}}\right)
\end{gathered}
$$

Where $\lambda$ is the detection threshold and $\gamma_{\mathrm{k}}$ is the signal to noise ratio.

$$
\lambda=\sigma_{w, k}^{2}\left(1+\frac{Q^{-1}\left(P_{F A}\right)}{\sqrt{N_{f} N_{t}}}\right)
$$

\subsection{Eigen Value Detector}

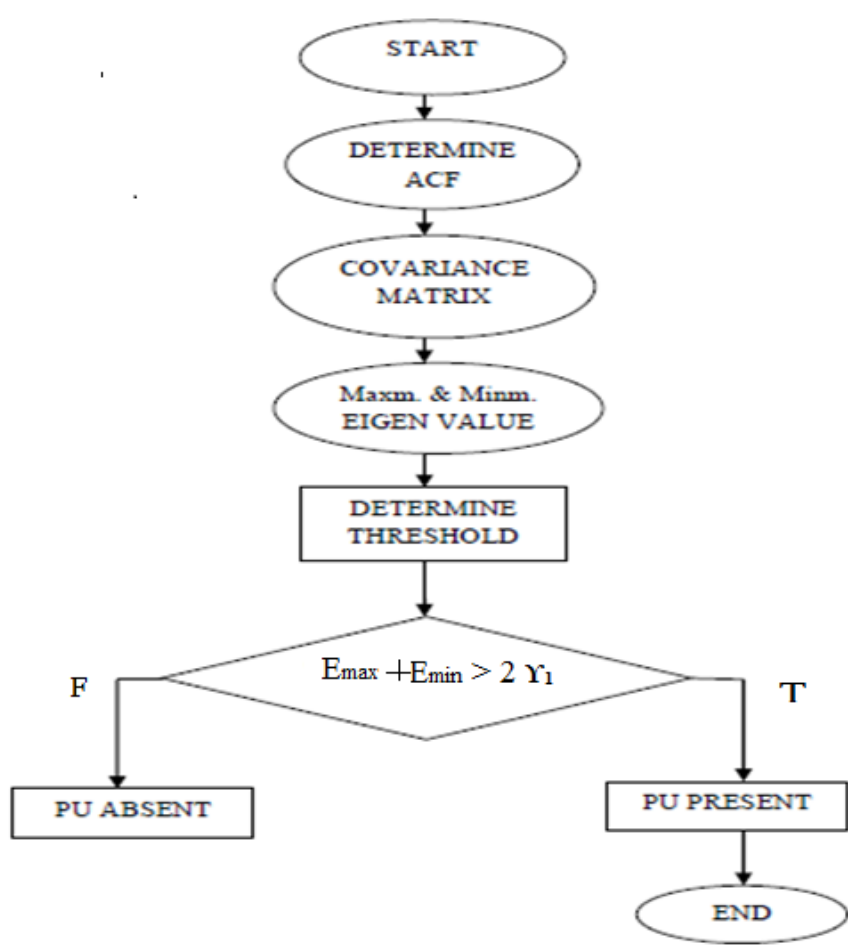

Figure 3: Block diagram of Eigen value detector

Signal detection is the fundamental problem in cognitive radio. The average of maximum and minimum Eigen value can be used to detect the signal existence in Eigen value detector (EVD) Based on Random Matrix Theorem (RMT) $\mathrm{P}_{\mathrm{FA}}$ is calculated. This method overcomes the noise uncertainty problem. The method can be used for various sensing applications without knowledge of the signal, channel and noise power [4]

\subsubsection{Algorithm}

Step1. Calculate the sample covariance matrix of the received signal.

Step2. Find the maximum and minimum Eigen value of covariance matrix.

Step3. Compute average of maximum and minimum Eigen value.

Step4. If average > threshold then signal exist, otherwise not exist.

The performance measuring parameters are given by

$$
\begin{gathered}
P_{f a}=1-F_{1}\left(\frac{\gamma_{1}\left(\sqrt{N_{s}}-\sqrt{M L}\right)^{2}-\mu}{\nu}\right) \\
P_{d}=1-F_{1}\left(\frac{\gamma_{1} N_{s}+N_{s}\left(\gamma_{1} \rho_{M L}-\rho_{1}\right) / \sigma_{\eta}^{2}-\mu}{\nu}\right)
\end{gathered}
$$

Where $\mathrm{N}_{\mathrm{s}}$-number of samples, M-sampling factor, Lsmoothing factor, $F_{1}$ is Tracy Widom Distribution function, $\mu$ is mean and $\mathrm{v}$ is variance. Threshold value is

$$
\gamma_{1}=\frac{\left(\sqrt{N_{s}}+\sqrt{M L}\right)^{2}}{\left(\sqrt{N_{s}}-\sqrt{M L}\right)^{2}}\left(1+\frac{\left(\sqrt{N_{s}}+\sqrt{M L}\right)^{-2 / 3}}{\left(N_{s} M L\right)^{1 / 6}} F_{1}^{-1}\left(1-P_{f a}\right)\right)
$$

\section{Volume 5 Issue 6, June 2016 www.ijsr.net}




\section{International Journal of Science and Research (IJSR) \\ ISSN (Online): 2319-7064 \\ Index Copernicus Value (2013): 6.14 | Impact Factor (2015): 6.391}

Threshold is not related to noise power; it can be precalculated based only $\mathrm{N}_{\mathrm{s}}, \mathrm{L}, \mathrm{P}_{\mathrm{fa}}$ irrespective of signal and noise.

\section{Simulation Results}

Mat lab R2013a is used as the platform to perform this task. Consider a random signal, which is the primary user signal shown in figure 4. It is then passed through an AWGN channel and resulted signal is the secondary user signal shown in figure 5. The number of samples is taken as 1000 .

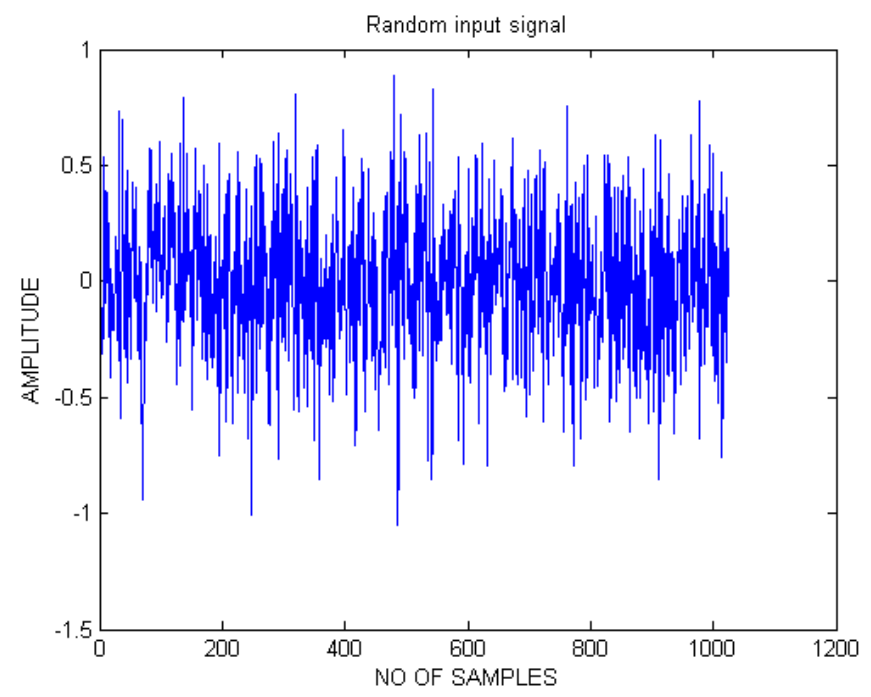

Figure 4: Random primary user signal

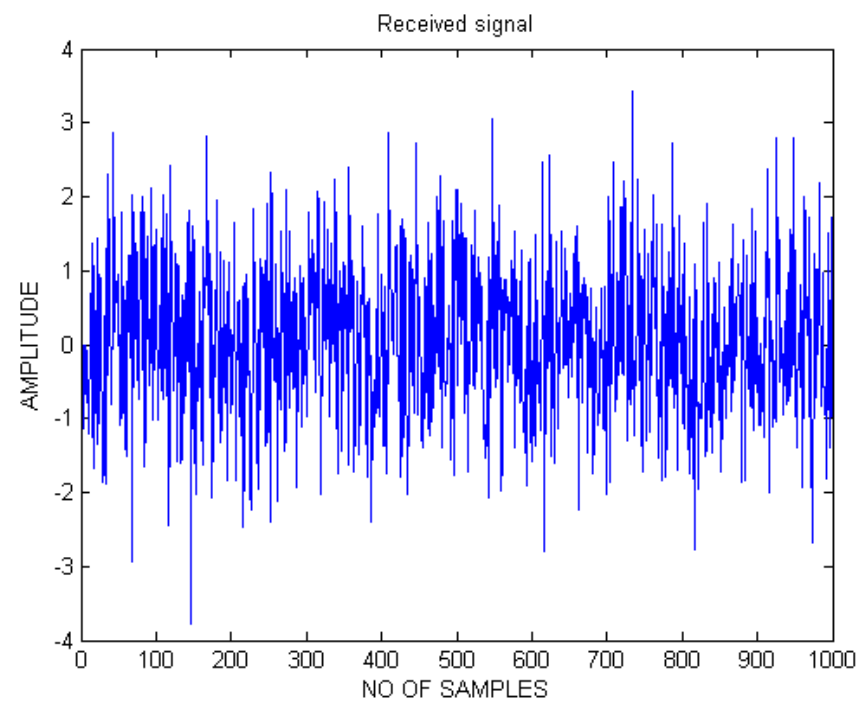

Figure 5: Received signal of secondary user

Spectrum sensing performance is evaluated by considering the probability of false alarm $\left(\mathrm{P}_{\mathrm{FA}}\right)$ and probability of detection $\left(\mathrm{P}_{\mathrm{D}}\right)$ parameters. The sensing performance is good when $\mathrm{P}_{\mathrm{D}}$ is high and $\mathrm{P}_{\mathrm{FA}}$ is low.

Figure 6 shows the SNR versus $\mathrm{P}_{\mathrm{D}}$ graph for energy detector and hybrid detector. At low SNR energy detector has poor performance. The detection probability of hybrid detector is higher than energy detector. At low SNR $P_{D}$ value of hybrid detector is higher, but for energy detector $\mathrm{P}_{\mathrm{D}}$ is very low.
Figure 7 shows the false alarm probability versus missed detection probability graph. $\mathrm{P}_{M}=1-\mathrm{P}_{\mathrm{D}}$, so as $\mathrm{P}_{\mathrm{D}}$ increases $\mathrm{P}_{\mathrm{M}}$ decreases. It is also shown that energy detector has poor performance and hybrid detector has better spectrum sensing performance.

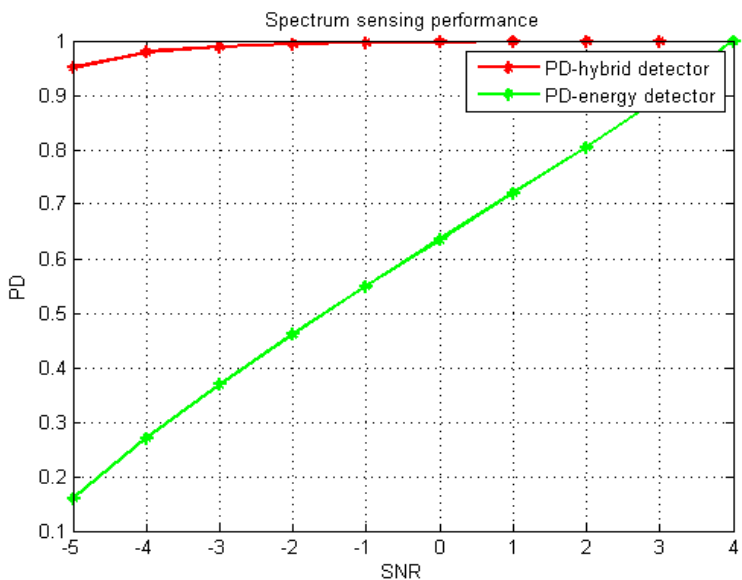

Figure 6: SNR Vs $\mathrm{P}_{\mathrm{D}}$ graph showing spectrum sensing performance of different detection schemes.

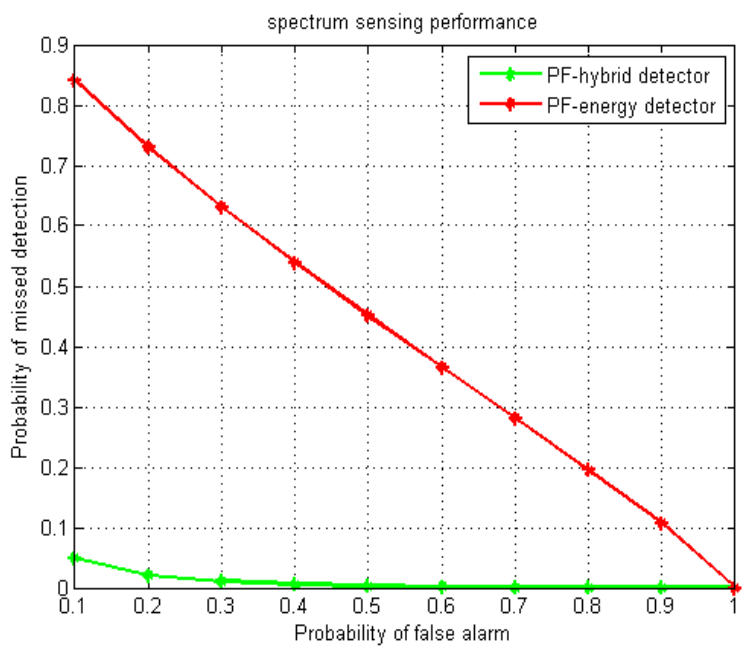

Figure 7: $\mathrm{P}_{\mathrm{FA}}$ Vs $\mathrm{P}_{\mathrm{M}}$ graph showing spectrum sensing performance of different sensing schemes.

The sensing capacity of energy detector and hybrid detector increases with increase in SNR. Capacity is higher for hybrid detection scheme.

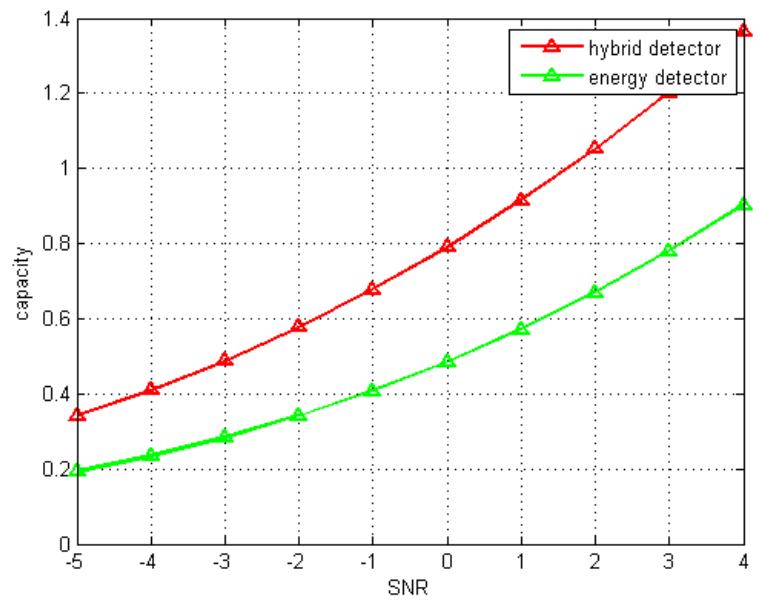

Figure 8: Capacity graph of energy detector and hybrid detector. 


\section{International Journal of Science and Research (IJSR) \\ ISSN (Online): 2319-7064 \\ Index Copernicus Value (2013): 6.14 | Impact Factor (2015): 6.391}

The received signal SNR value and the corresponding detection methods are shown by the table 1 . The threshold value for the hybrid detector ie, $\lambda_{H}$ is set as 10.5. Then each SNR value is compared with this threshold value. If SNR is higher than threshold, energy detector is performed well. Otherwise Eigen value detector is performed the spectrum sensing phenomena. Since the ED highly depends on the noise values, it is not a good method of spectrum sensing under low SNR values [5].

Table 1: SS method used for each SNR value

\begin{tabular}{|c|c|}
\hline Received signal SNR & SS Method \\
\hline 10.3860 & EVD \\
\hline 10.3489 & EVD \\
\hline 10.6813 & ED \\
\hline 10.5466 & ED \\
\hline 10.0818 & EVD \\
\hline 10.4980 & EVD \\
\hline 10.1667 & EVD \\
\hline 10.3403 & EVD \\
\hline 10.8346 & ED \\
\hline 9.9099 & EVD \\
\hline
\end{tabular}

\section{Conclusion}

Cognitive radio is an effective approach for better sharing the underutilized communication spectrum. Different spectrum sensing methods are introduced in order to achieve better utilization of the spectrum. The energy detection is the most popular SS method in CR systems. The main limitation of energy detector is its inherent sensitivity to the knowledge of noise variance. It will affect the overall spectrum sensing performance and increases the complexity of the system. The Eigen value based detector doesn't depend on the knowledge of noise information. So in order to improve the SS performance a Hybrid detection method can be used. It uses a combination of energy detector and Eigen value based detection. This hybrid detector is a threshold based detector. This will improve the SS performance of cognitive radio.

\section{Acknowledgment}

I would like to express profound gratitude to our Head of the Department, Prof. Cherian Schariah, for his encouragement and for providing all facilities for my work. I express my highest regard and sincere thanks to my guide, Asst. Prof. Ms. Sangeeta T.R., who provided the necessary guidance and serious advice for my work.

\section{References}

[1] S. Haykin, "Cognitive radio: Brain empowered wireless communications," IEEE J. select. Areas commn., vol. 23, pp.201-220 Feb. 2011.

[2] R. Tandra and A Sahai "SNR walls for signal detection," IEEE J.Sel. Topics signal process, vol. 2, pp.2124.Jan. 2007.

[3] Y.Zeng and Y. C. Liang "Eigen value based spectrum sensing algorithms for cognitive radio," IEEE Tras. Vol.57, pp.17841793, Jun. 2009.

[4] A.Kortun, T. Ratnarajah, M. Sellathurai and C. B. Papadias,"On the performance of eigen value based cooperative spectrum sensing for cognitive radio," IEEE J. Sel. Topics signal process, vol. 5, pp.4955,Feb. 2011.

[5] F. Khan and K. Nakagawa "Comparative study of spectrum sensing techniques in cognitive radio networks,", Computer and Information Technology (WCCIT), pp.1-8,2013.

[6] www.mathworks.in

\section{Author Profile}

Chinchu S received the B-Tech degrees in Electronics and Communication Engineering from M.G University, Kerala at Sree Buddha college of Engineering for women in 2013. And now she is pursuing her $\mathrm{M}$-Tech degree in Communication Engineering under the same university in Sree Buddha college of Engineering for women.

Sangeeta T R working as Assistant Professor in department of Electronics and Communication, Sree Buddha college of Engineering for women, Elavumthitta, Pathanamthitta. 